[This document contains the author's accepted manuscript. For the publisher's version, see the link in the header of this document.]

\title{
Application of Uncertain Reasoning to Business Decisions: An Introduction
}

\author{
By Prakash P. Shenoy and Rajendra P. Srivastava \\ The University of Kansas School of Business
}

\section{Paper citation:}

Srivastava, Rajendra. (2003) Application of Uncertain Reasoning to Business Decisions: An Introduction. Information Systems Frontiers, 5 (4), 343-344. 


\title{
Application of Uncertain Reasoning to Business Decisions: An Introduction
}

\author{
Prakash P. Shenoy and Rajendra P. Srivastava \\ University of Kansas School of Business
}

We are very pleased to serve as guest editors for this special issue of Information Systems Frontiers on business applications of uncertain reasoning. We would like to thank the chief editors Raghav Rao and Ram Ramesh for providing this opportunity. We would also like to thank the reviewers, Kurt Fanning, Rolf Haenni, Juerg Kohlas, Ryan Labrie, Paul-Andre Monney, Jim Nelson, Dan Oleary, Raghu Santanam, Benjamin Shao and Philippe Smets, for their thoughtful and constructive reviews. Finally we would like to express our sincere thanks to the authors for their contributions. Without their contribution this issue would not have been possible.

Uncertain reasoning, an integral part of information systems, is a decision support modeling tool that has taken on increasing importance in the recent past. It has been utilized in applications as diverse as credit ratings, medical diagnosis, multi-sensor fusion, and aggregating audit evidence. This special issue focuses on the business aspects of uncertain reasoning and brings a cross-disciplinary awareness to the business community.

This special issue contains four papers. The first paper titled "A Comparison of Bayesian and Belief Function Reasoning" is by Cobb and Shenoy. The authors start the paper by providing the basic backgrounds on the two uncertainty calculi, Bayesian probability theory and belief function theory, especially regarding their representations, semantics, and calculi for combining and marginalizing uncertainties associated with the 
propositions in the problem domain. Traditionally, Bayesian probability framework has been used for business decisions, especially when the probability distributions in the problem domains are known. Belief-function theory, on the other hand, has been used for aggregating evidence, especially when there is partial ignorance about the problem domain, e.g., the prior probability distribution for some of the variables may not be known. Ignorance or partial ignorance is quite common in business disciplines whether it is accounting, finance or management science. It is commonly believed that belief function theory is more expressive than Bayesian probability theory since Bayesian probability theory is a special case of belief function theory. Cobb and Shenoy argue otherwise. They highlight the similarities and differences between the two calculi and argue that both calculi have roughly the same expressive power. Once a problem is modeled in one calculus, one can transform it into the other by means of a suitable transformation. They use a "real world" example from the sensor fusion domain to demonstrate their claim.

The second paper titled "Applications of belief functions in business decisions: A review" by Srivastava and Liu provides a review of the literature on applications of Dempster-Shafer theory of belief functions (DST) in business decisions. Here, the authors show that DST is a better framework for mapping uncertainties in the application domains than Bayesian theory of probabilities. First part of the paper provides a review of the literature on the applications of DST to auditing problems and discusses the approaches to dealing with three kinds of evidence, namely, hierarchical evidence, versatile evidence, and statistical evidence. The authors also review the literature on judgment under ambiguity and business model combination using DST. Finally, the 
authors provide a discussion on the application of theory of linear belief functions, a new extension of DST, and demonstrate its application through an example.

The third paper is titled "A Set Theoretical Foundation of Qualitative Reasoning and Its Application to the Modeling of Economics and Business Management Problems" and is written by Hinkkanen, Lang, and Whinston. As the title suggests, this paper deals with qualitative reasoning in which problems are formulated as qualitative constraints and are analyzed using a qualitative calculus. Their calculus is based on set theory and is motivated by some types of problems in management sciences. They emphasize the significance of discrete, dynamic and optimization models in business management and economics.

The last paper basically deals with predicting corporate acquisitions using rule induction approach with uncertain knowledge. This paper is written by Ragothaman, Naik, and Ramakrishnan. The authors first provide a review of the literature relevant to corporate acquisitions and then discuss the knowledge acquisition technique in term of IFTHEN production rules using a commercially available rule induction technique called, Induction on Extremely Large (IXL) databases. They also discuss how IXL manages uncertainties through confidence factors. Based on the rules generated by IXL using a set of training data comprising of 65 acquired and 65 non-acquired firms, the authors develop a prototype rule based expert system called ACQTARGET. They use a separate holdout sample of 32 acquired and 32 non-acquired firms to validate the expert system results. Also, they compare the performance of the expert system, ACQTARGET, with a conventional discriminant analysis model and a logit model using the same data. Their results show that their expert system performs as well as the statistical models. 